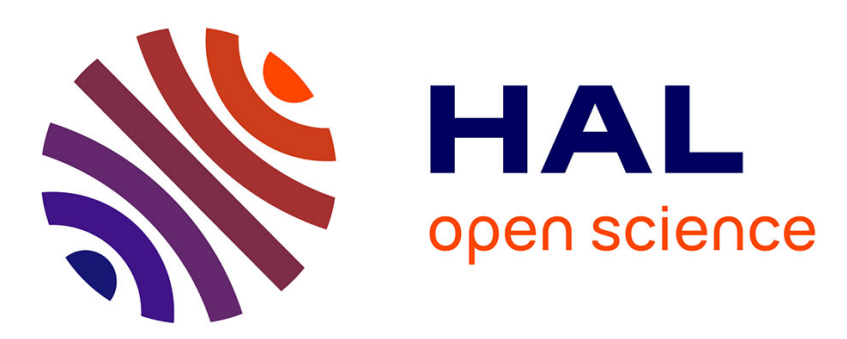

\title{
Souvenirs from ECAL 2017: create, play, experiment, discover - revealing the experimental power of virtual worlds
}

Carole Knibbe

\section{To cite this version:}

Carole Knibbe. Souvenirs from ECAL 2017: create, play, experiment, discover - revealing the experimental power of virtual worlds. Adaptive Behavior, 2018, 26 (1), pp.37-40. hal-01952933

\section{HAL Id: hal-01952933 \\ https://hal.inria.fr/hal-01952933}

Submitted on 12 Dec 2018

HAL is a multi-disciplinary open access archive for the deposit and dissemination of scientific research documents, whether they are published or not. The documents may come from teaching and research institutions in France or abroad, or from public or private research centers.
L'archive ouverte pluridisciplinaire HAL, est destinée au dépôt et à la diffusion de documents scientifiques de niveau recherche, publiés ou non, émanant des établissements d'enseignement et de recherche français ou étrangers, des laboratoires publics ou privés. 


\title{
Souvenirs from ECAL 2017: Create, play,
}

\section{experiment, discover - revealing the experimental power of virtual worlds}

\author{
Carole Knibbe ${ }^{1,2}$ \\ ${ }^{1}$ Inria, France \\ ${ }^{2}$ Univ Lyon, INSA-Lyon, CarMeN laboratory, INSERM U1060, \\ F-69621, Lyon, France \\ carole.knibbe@inria.fr
}

ECAL 2017, the Fourteenth European Conference on Artificial Life, was held September 4-8th 2017, in Lyon, France (https://project.inria.fr/ ecal2017/). Since the first ECAL in 1991, the conference is the main international event of the International Society for Artificial Life (http://alife.org/) in odd-numbered years, alternating with ALife, the International Conference on the Synthesis and Simulation of Living Systems. This edition gathered two hundred participants, coming from Europe of course, but also from America, Asia, Africa and Oceania.

Seven plenary talks have been presented, covering a wide range of artificial life topics, related to the conference theme - "Create, play, experiment, discover: revealing the experimental power of virtual worlds". Indeed, one goal of the conference was to investigate the place of the experimental approaches within the artificial life field, both in terms of how we can use experimental results from chemistry, biology or social sciences, and in terms of how we experiment with our own artefacts. 


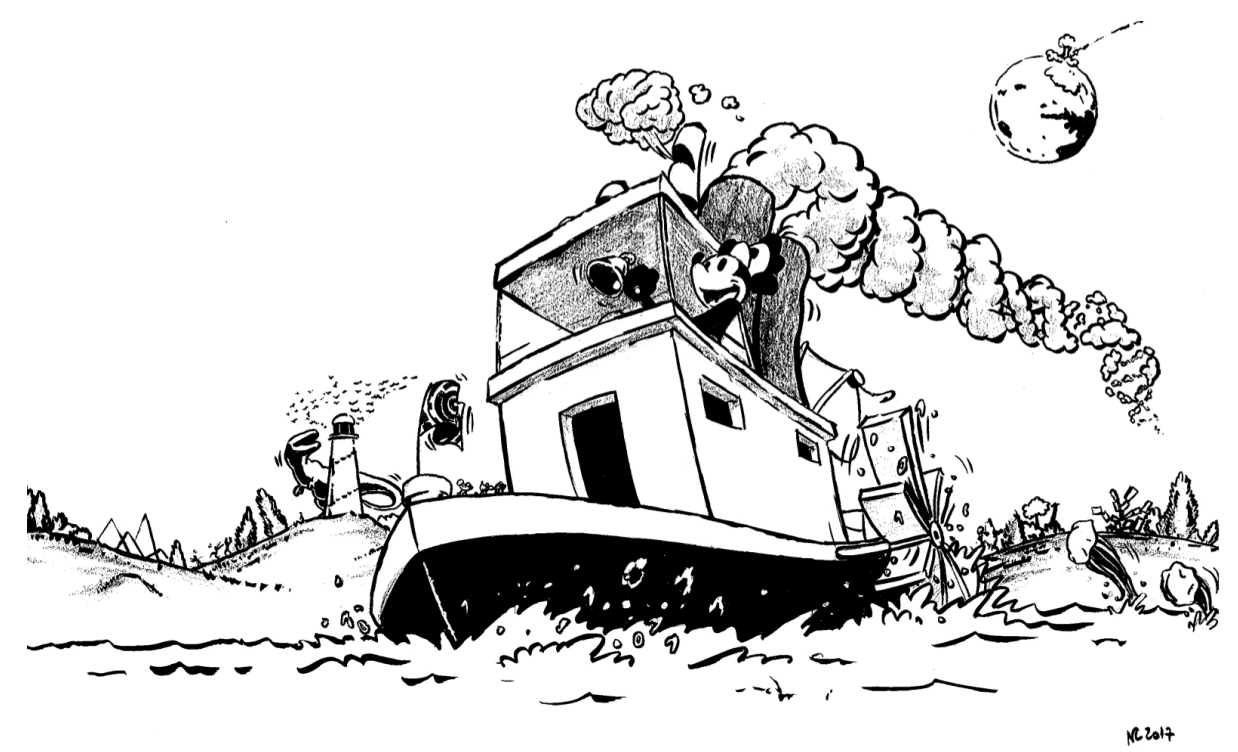

Figure 1: ECAL 2017 cartoon by Nicolas Comte. 15 elements related to ECAL 2017 keynotes, topics and events are hidden in the cartoon, will you be able to find them all?

André Brack, an astrobiologist from the Centre de Biophysique Moléculaire in Orléans, France, opened the conference with an introductory lecture on the chemical "automata" at the origin of life. With a communicative enthusiasm, he presented the insights yielded by the last space missions, on the possible sources of prebiotic carbon for example. He also introduced us to dilemma faced by chemists like singularity (very specific local conditions, allowing for a few births of life seeding the whole primitive ocean) vs ubiquity (widespread conditions allowing for spontaneous births of life everywhere). Another one is about the best method to try and recreate the origin of life: "classical", step-by-step predictive chemistry, or alchemist-style stochastic chemistry? The latter is a new approach developed in Orléans, where one submits a maximum of prebiotic ingredients under prebiotic conditions and lets the system run for months.

Bill Sellers, a computational zoologist from University of Manchester, United Kingdom, made an exciting lecture on synthetic palaeontology, or how to reconstruct ancient life by designing virtual robots. Bill insisted on the importance of 
calibrating the simulations with ground-truth data, which he obtained by filming animal locomotion in zoos with a specific video photogrammetry system. He also stressed that multi-goals and multi-physics simulations are in order for better simulation outcomes. Indeed, when reconstructing locomotion, it is generally assumed that the animal is either attempting to maximise economy or speed, but in reality "the locomotor system in most animals is not a single function organ and it has to cope with a range of performances and their associated control and mechanical issues" (Sellers, 2017). In a memorable example, he showed us that contrary to what was shown in the Jurassik Park movie, Tyrannosaurus rex likely could not run, because running would have generated unacceptably high skeletal loads (Sellers et al., 2017).

Philippe Faure, a neurophysiologist from Université Pierre et Marie Curie, Paris, France, presented the original experimental approaches he develops in his lab to investigate the decision making process in animals and the underlying neurobiological mechanisms. He presented for example "Souris City", a socially enriched setup where mice are housed in larger groups than in standard conditions, and where they can explore several connected compartments, resembling the natural living conditions of mice. Such a setting allows to gather information on the hierarchical structure within a group, for example. Philippe also presented his recent results on the molecular basis of decision-making with uncertain rewards. By combining experiments and computational modeling, he showed that wild-type mice are motivated to explore zones with uncertain rewards (while mutant mice lacking a part of the nicotinic acetylcholine receptor are less inclined to do so). Those results are "consistent with theoretical work on optimal exploration and intrinsic motivation. In this view, exploration and exploitation are entangled: uncertainty is given a value that can be compared to and added to the value of primary rewards" (Naudé et al., 2016).

Csaba Pál, a molecular evolutionary biologist from the Biological Research Centre of the Hungarian Academy of Sciences, Szeged, Hungary, addressed the critical question of how complex adaptations can evolve. He argued that com- 
plex adaptations are prevalent in molecular systems, with for example the evolution of new protein functions involving multiresidue interactions or the evolution of multi-step metabolic pathways. He presented the latest insights brought by experimental evolution and systems biology. Protein evolution studies, for instance, do not support the non-adaptive theory that neutral or slightly deleterious mutations would prepare the ground for later beneficial mutations that lead to new, beneficial traits. An alternative, adaptive scenario is that complex molecular traits can readily evolve through series of adaptive steps if the environment is dynamically changing (Pál, 2017).

The lecture of Sabine Hauert, a roboticist from University of Bristol, United Kingdom, addressed the problem of scaling to large numbers of robots or nanoparticles in swarm engineering. We learned that scaling to thousands of robots requires shifting paradigm in the way swarm behavior is designed. She showed us how, by removing assumptions regarding precise control of robot motion, it is possible to deploy swarm behaviors on large amounts of uncalibrated robots performing random motion and simple local exchanges of information (Crosscombe et al., 2017). When swarm engineering is used in the context of cancer treatment by nanoparticles, the problem of scaling is even more acute, since it typically involves trillions of particles without a controller. Predicting and engineering these collective behaviors is often counterintuitive. To help researchers develop a working intuition of these systems, she developed Nanodoc, a game to crowd-source nanomedicine, where users can see the simulated behaviour of nanoparticles in a virtual tumour and iteratively design or tweak the nanoparticle system dynamics (Hauert, 2017).

In a lecture directly related to the conference theme, Viola Schiaffonati, a philosopher of science from Politecnico di Milano, Italy, told us how experimenting with computing and in computing requires to stretch the traditional notion of controlled experiment. She introduced the notion of exploratory experiments, where simulation results are not only used to test or reject a theory, but also used to shape the theoretical model supporting them. By investigating 
autonomous robotics in particular, she found that such exploratory experiments can be "driven by the desire of investigating the realm of possibilities pertaining to the functioning of an artefact and its interaction with the environment in the absence of a proper theory or theoretical background. So hypotheses cannot be clearly stated and, even if the ultimate goal is to acquire knowledge about the performance of the artefacts under investigation and to find out proper concepts to formulate possible regularities, the experimenter is not in full control of the experimental setting due to the impossibility of anticipating all the plausible outcomes. Therefore, when experiments are explorative, control should be intended in a posteriori form, in opposition to the a priori form of the traditional experimental contexts. (...) [With exploratory experiments] the possibility of full anticipation disappears and control is in part carried out after the artefact has been inserted into society" (Schiaffonati, 2017).

Last but not least, and because artificial life and art often inspire each other, Andreas Wessel-Therhorn, an animator and film director, and Laurent PujoMenjouet, a biomathematician and Disney enthusiast from Université Lyon 1, France, introduced us to the animation principles used to give the illusion of life. In a lecture that turned into a show with live demos, Andreas and Laurent walked us through the twelve principles established by Disney's "Nine Old Men" in the 1930s (Thomas and Johnston, 1981). These principles - squash and stretch, anticipation, staging, straight ahead action and pose to pose, follow through and overlapping action, arcs, slow in and slow out, secondary action, timing, exaggeration, solid drawing, appeal - are still the basis for Western character animation today. Andreas and Laurent asked whether such principles could be used in robotics to build robots able to give the illusion of life in such a way that we could feel sympathetic to them.

With its 64 contributed talks (for a total of 131 submissions) and 23 contributed posters, ECAL showed the diversity of the Artificial Life community. Indeed, these contributions covered all the topics of Artificial Life, the most represented being (i) Evolutionary dynamics, (ii) Complex dynamical sys- 
tems and networks, (iii) Perception, cognition and behavior and (iv) Bioinspired robotics and embodied systems. The best paper award was granted to Nick Moran and Jordan Pollack, for their work on the "Effects of Cooperative and Competitive Coevolution on Complexity in a Linguistic Prediction Game" (Moran and Pollack, 2017). The best poster award was granted to Peter Aaser and collaborators, for their work on "Towards Making a Cyborg: A ClosedLoop Reservoir-Neuro System" (Aaser et al., 2017). This clearly illustrates the intricacy between ALife and SAB topics and communities... The whole proceedings (Knibbe et al., 2017) are available in open access at MIT Press (http://cognet.mit.edu/journal/ecal2017).

In addition, six workshops and ten tutorials have been organized during the conference, the diversity of the topics illustrating the vitality of the field:

- Workshops:

- Evolution of Physical Systems,

- Morphogenic Engineering,

- Social Learning and Cultural Evolution,

- What can Synthetic Biology offer to (Embodied) Artificial Intelligence (and vice versa)?

- Agency In The Physical Sciences,

- Living Architecture.

- Tutorials:

- ISAL School,

- Using MABE (Modular Agent Based Evolution),

- Building Artificial Life Systems for the Web with Empirical,

- Evolutionary Game Theory: Models and Applications,

- Evolutionary Robotics - A practical guide to experiments with real hardware, 
- Evolution In The Cloud,

- Simulating Complex Systems with FLAME GPU,

- Avida-ED: a web-based, GUI implementation of the Avida software platform, for educational use,

- Digital Coevolution: a beginners approach,

- Evolution of Neural Networks.

During the conference dinner on the Hermes boat on the Rhône and Saône rivers, the 2017 ISAL awards were granted to Jordan B. Pollack (Lifetime Achievement in the field of Artificial Life), Jean-Baptiste Mouret (Distinguished Young Investigator in the field of Artificial Life), Kenneth O. Stanley and Risto Miikkulainen (Outstanding Paper of the Decade 2002 - 2012), Richard A. Watson and Eörs Szathmáry (Outstanding Paper of 2016 in the field of Artificial Life), Rob Pennock (Education and Outreach using Artificial Life) and René Doursat (Exceptional Service to the Artificial Life Community).

Another memorable moment of this cruise was Steen Rasmussen sharing anecdotes and memories of three decades of artificial life conferences. Indeed, 2017 marks the 30th anniversary of the first Artificial Life Workshop, held at Los Alamos National Laboratory in 1987. 2017 also happened to be the ultimate edition of ECAL, this series of fourteen conferences held in Paris, Brussels, Granada, Brighton, Lausanne, Prague, Dortmund, Canterbury, Lisbon, Budapest, Taormina, York and finally Lyon. But this closure is not an end. The two alternating conferences, ECAL and Alife, will be merged in a single event, to be held every year. The end of ECAL is a new starting point for the community. Indeed, as one can immediately see it when looking at the conference proceedings, by its contributors, by its audience or by its scientific committee, ECAL was not an exclusively European event. It was a world-renowned conference series that attracted the best contributions in its field every other year, like ALife did in alternation. Hence the fusion of both events in a single format is just logical: Let us meet again in Tokyo at the 2018 Conference on Artificial 
Life, a hybrid of the European Conference on Artificial Life (ECAL) and the International Conference on the Synthesis and Simulation of Living Systems (ALife)!

\section{Acknowledgments}

As the chair of the conference the author would like to thank all the institutional sponsors of ECAL 2017 (INRIA, CNRS, INSA-Lyon, Université Claude Bernard Lyon 1, Université Pierre et Marie Curie, Fédération Informatique de Lyon, Institut Rhône-Alpin des Systèmes Complexes), as well as the members of the program committee and the members of the ISAL board for their invaluable help. Needless to say, organizing a conference like ECAL requires lot of work, and this conference would not have been a success without the precious help of the organisation committee and numerous volunteers that I would like to warmly thank here: Guillaume Beslon, David Parsons, Dusan Misevic, Jonathan Rouzaud-Cornabas, Nicolas Bredèche, Salima Hassas, Olivier Simonin, Hédi Soula, Vincent Liard, Nicolas Comte, Charles Rocabert, Priscila Biller, Laurent Turpin, Caroline Lothe, Sophie Azzaro, Nicolas Nadisic and Audrey Denizot.

\section{References}

Aaser, P., Knudsen, M., Ramstad, O. H., van de Wijdeven, R., Nichele, S., Sandvig, I., Tufte, G., Bauer, U. S., Halaas, Ø., Hendseth, S., Sandvig, A., and Valderhaug, V. (2017). Towards making a cyborg: A closed-loop reservoirneuro system. In Knibbe et al. (2017), pages 430-437.

Crosscombe, M., Lawry, J., Hauert, S., and Homer, M. (2017). Robust distributed decision-making in robot swarms: Exploiting a third truth state. In 2017 IEEE/RSJ International Conference on Intelligent Robots and Systems, IROS 2017, Vancouver, BC, Canada, September 24-28, 2017, pages 4326-4332. IEEE. 
Hauert, S. (2017). Swarm engineering across scales: From robots to nanomedicine. In Knibbe et al. (2017), pages 11-12.

Knibbe, C., Beslon, G., Parsons, D. P., Misevic, D., Rouzaud-Cornabas, J., Bredèche, N., Hassas, S., Simonin, O., and Soula, H., editors (2017). Proceedings of the Fourteenth European Conference Artificial Life, ECAL 2017, Lyon, France, September 4-8, 2017. MIT Press.

Moran, N. and Pollack, J. B. (2017). Effects of cooperative and competitive coevolution on complexity in a linguistic prediction game. In Knibbe et al. (2017), pages 298-205.

Naudé, J., Tolu, S., Dongelmans, M., Torquet, N., Valverde, S., Rodriguez, G., Pons, S., Maskos, U., Mourot, A., Marti, F., and Faure, P. (2016). Nicotinic receptors in the ventral tegmental area promote uncertainty-seeking. Nature Neuroscience, 19(3):471-478.

Pál, C. (2017). Evolution of complex adaptations. In Knibbe et al. (2017), pages $6-7$.

Schiaffonati, V. (2017). Experimenting with computing and in computing: Stretching the traditional notion of experimentation. In Knibbe et al. (2017), pages $2-3$.

Sellers, W. I. (2017). Synthetic palaeontology: Reconstructing ancient life using virtual robots. In Knibbe et al. (2017), pages 8-9.

Sellers, W. I., Pond, S. B., Brassey, C. A., Manning, P. L., and Bates, K. T. (2017). Investigating the running abilities of Tyrannosaurus rex using stressconstrained multibody dynamic analysis. PeerJ, 5:e3420.

Thomas, F. and Johnston, O. (1981). Disney animation: The illusion of life. Abbeville Press, New York, 6 edition. 


\section{About the Author}

Carole Knibbe is an Associate Professor in Computational Biology at INSA Lyon (National Institute for Applied Sciences), Lyon, France. She builds mathematical and computational models of biological systems, with applications in molecular evolution, and, more recently, in lipid metabolism. She is the head of the Bioformatics and Modelling master program of INSA Lyon. She also served as scientific chair for ECAL 2017, the Fourteenth European Conference on Artificial Life. 